\title{
TIME DOMAIN METHOD FOR THE DETERMINATION OF THE STEADY STATE BEHAVIOUR OF NONLINEAR CIRCUITS DRIVEN BY MULTI-TONE SIGNALS
}

\author{
A. Brambilla \\ Dipartimento di Elettronica e Informazione, Politecnico di Milano, Milano, Italy \\ brambill@elet.polimi.it \\ G. Storti-Gajani \\ Dipartimento di Elettronica e Informazione, Politecnico di Milano, Milano, Italy
}

DOI: 10.36724/2664-066X-202I-7-3-12-16

\begin{abstract}
Time domain methods, while well suited to compute the steady state behaviour of strongly nonlinear non-autonomous electrical circuits, are inefficient if the periods of the forcing signals have a very large minimum common multiple. The solution of the periodicity constraint requires to integrate the differential algebraic equation (DAE) describing the circuit along the $\mathrm{T}$ period and this can be a CPU time consuming task. Literature reports several attempts to extend the $\mathrm{SH}$ method to simulate circuits driven by multi-tone signals [2] [4] [5]. However, as far as we know, all they suffer of limitations and it is our opinion that an efficient and general extension has not been found, yet. In this paper we present a possible extension that takes its origin from the previous approach reported in [2]. In this paper a modification of the conventional shooting method is presented that tries to overcome the above drawback.
\end{abstract}

KEYWORDS: shooting method, harmonic balance, steady state analysis 


\section{INTRODUCTION}

The harmonic balance method (HB) and the shooting one ( $\mathrm{SH})$ are largely used to numerically determine the steady state solution of autonomous and nonautonomous circuits. One main difference between HB and $\mathrm{SH}$ is that the former can be adopted to simulate non-autonomous circuits driven by periodic signals whose periods can be very different or even noncommensurate, while, in general, the application of $\mathrm{SH}$ to these circuits is convenient only when the minimum common multiple ( $\mathrm{mcm}$ ) of the periods of the driving signals, denoted by $T$, is a small integer multiple of each of them. This limit of the SH method is due to the fact that its formulation is based on the solution of the periodicity constraint $y_{s}(T+t)-y_{s}(t)=0, \forall t>t_{0}$ where $y_{s}(t)$ is the steady state solution and $t$ is time. The solution of the periodicity constraint requires to integrate the differential algebraic equation (DAE) describing the circuit along the $T$ period and this can be a CPU time consuming task.

Literature reports several attempts to extend the SH method to simulate circuits driven by multi-tone signals [2] [4] [5]. However, as far as we know, all they suffer of limitations and it is our opinion that an efficient and general extension has not been found, yet.

In this paper we present a possible extension that takes its origin from the previous approach reported in [2]. Even though the proposed approach solves some of the drawbacks evidenced in [2], it is still far to be a general method. A main restriction is that it can be applied only to a specific class of circuits that satisfy the assumption that the steady state solution $y_{s}(t)$ has a spectrum characterised by distinct and suitably separated bands. In this case, we show that through an excursus in the frequency domain it is possible to reformulate the periodicity constraint, so that $y_{s}(t)$ can be computed only along a small set of time windows each of length $\Delta$ and evenly spaced in the circuit working period $T$. We show that the length $\Delta$ of the time window can be chosen as a small fraction of $T$ thus allowing an efficient application of the SH method. Despite the above mentioned restriction, we believe that the proposed method has practical interest, in fact a large number of important circuits for RF applications such as for example mixers, modulators, large band RF amplifiers and travelling waveform amplifiers, belong to this class.

\section{Problem formulation}

Consider the state equation

$$
\left\{\begin{array}{l}
\frac{d y}{d t}(t)+f(y(t), t)=0 \quad t \geq t_{0} \\
y\left(t_{0}\right)=y_{0}
\end{array}\right.
$$

where $y(t): \mathbb{R} \rightarrow \mathbb{R}^{\mathbb{N}}$ is suitably differentiable, $f(y(t), t): \mathbb{R}^{\mathbb{N}+\mathbb{1}} \rightarrow \mathbb{R}^{\mathbb{N}}$ embeds the periodic driving signals, $t \in \quad+$ is time and $y_{0} \in \mathbb{R}^{\mathbb{N}}$ is the value of the initial condition at the $t_{0}$ time instant. Suppose that (1) admits the $y_{s}(t): \mathbb{R} \rightarrow \mathbb{R}^{\mathbb{N}}$ steady state solution represented by the series

$$
y_{s}(t)=\sum^{\infty}\left(\varrho_{p} e^{j p \omega t}-\varrho_{p}^{*} e^{-j p \omega t}\right)
$$

where $\omega=2 \pi / T$, being $T$ the period of the steady state solution and $\varrho_{p} \in \mathbb{C}^{\mathbb{N} \times \mathbb{N}}$ are matrices whose entries are the coefficients of the Fourier series and * represents complex conjugation. Being by definition $y_{s}(t)$ a solution of (1), we can write

$$
y(t)=y_{s}(t)+r(t)
$$

where the $r(t): \mathbb{R} \rightarrow \mathbb{R}^{\mathbb{N}}$ function describes how the solution at the current time instant $t$, that depends on the $y\left(t_{0}\right)$ initial condition, is far from the periodic solution $\widehat{y}_{s}$.

Our aim is to find the $\widehat{y}_{s}$ initial condition such that $y(t)$ satisfies the periodicity constraint

$$
y(t) \theta+T)-y(t)=0
$$

Adoption of the periodicity constraint (4) requires to integrate (1) at least along one $T$ period but this may require too much numerical effort. To this end we define a new periodicity constraint that solves the above drawback. From (3) we can write

$$
\begin{gathered}
y(t+\Delta)-y(t)=\sum_{p=0}^{\infty}\left(\varrho_{p} e^{j p \omega(t+\Delta)}-\varrho_{p}^{*} e^{-j p \omega(t+\Delta)}\right)- \\
\sum_{p=0}^{\infty}\left(\varrho_{p} e^{j p \omega t}-\varrho_{p}^{*} e^{-j p \omega t}\right)+r(t+\Delta)-r(t)=0
\end{gathered}
$$


where $\Delta \ll T$. We wish to find $y_{s}(t)$ such that $r(t+\Delta)-r(t)=0 \quad \forall t \geq t_{0}$. Consider the $W_{T}(\zeta(t), t)$ operator, where $\zeta(t): \mathbb{R} \rightarrow \mathbb{R}^{\mathbb{N}}$ is a generic function, that removes all components in frequency but only those of the spectrum of $y_{s}(t)$. The application of $W_{T}(\zeta(t), t)$ to $(5)$ leads to

$$
W_{0}(y(t+\Delta)-y(t), t)=W_{0}(r(t+\Delta)-r(t), t)=0
$$

For example $W_{T}(\zeta(t), t)$ can be chosen as

$$
W_{T}(\zeta(t), t)=\int_{t}^{t+T} \zeta(\tau) d \tau
$$

but a full $T$ period integration is still required so that it is useless to our purpose.

Introduce now the strong assumption that $\varrho_{p}=0$ for $0 \leq L<p<Q$ with $L \ll Q, L, Q \in \mathbb{N}$. This means that the spectrum of $y_{s}(t)$ is characterised by two distinct bands one laying in the frequency range $\left[0, \nu_{L}\right]$ and the other of possibly infinite bandwidth laying above $\nu_{Q}$. Consider the new version $W_{\Delta}(\zeta(t), t)$ of the $W_{T}(\zeta(t), t)$ operator that now zeroes all the components of the spectrum of $\zeta(t)$ laying above the $\nu_{\Delta}=1 / \Delta$ cut-off frequency

$$
W_{T}(\zeta(t), t)=\int_{t}^{t+\Delta} w(\tau, t) \zeta(\tau) d \tau
$$

where $w(\tau, t): \mathbb{R} \rightarrow \mathbb{R}^{\mathbb{N}}$ is a suitable windowing function. A good description of the various windowing functions can be found in [3]. Each of them can be considered as a comb filter whose zeroes are at frequencies multiple of $\nu_{\Delta}$.

This operator requires to integrate (1) only along the $\Delta$ time interval and if we chose $\Delta=T / q, q<Q$, this means that $\Delta$ can be a small fraction of $T$. The application of $W_{\Delta}(\zeta(t), t)$ to $(5)$ leads to

$$
\begin{gathered}
\overbrace{\sum_{p=0}^{L}\left(\varrho_{p} e^{j p \omega(t+\Delta)}-\varrho_{p}^{*} e^{-j p \omega(t+\Delta)}\right)}^{(\phi(y(t), t, t+\Delta)-y(t), t)=}- \\
\overbrace{\sum_{p=0}^{L}\left(\varrho_{p} e^{j p \omega t}-\varrho_{p}^{*} e^{-j p \omega t}\right)}^{\sigma_{1}(t)}+ \\
W_{\Delta}(r(t+\Delta)-r(t), t)=0
\end{gathered}
$$

The two $\sigma_{1}(t)$ and $\sigma_{2}(t)$ series are truncated at the $L$ index since the spectrum of $y_{s}(t)$ has been zeroed above $\nu_{q}$. Further they have identical coefficients and, as it can be seen, $\sigma_{1}(t)$ is a version of $\sigma_{2}(t)$ delayed by $\Delta$. In the following this aspect is exploited.

We chose $2 L+1$ evenly spaced samples denoted as $t_{0}, \ldots, t_{2 L}$ along the $T$ time interval and apply $W_{\Delta}(\zeta(t), t)$ to both $y\left(t_{n}+\Delta\right)$ and $y\left(t_{n}\right)$. Define

$$
z \equiv\left[\begin{array}{c}
W_{\Delta}\left(\phi_{z}\left(t_{0}\right), t_{0}\right) \\
W_{\Delta}\left(\phi_{z}\left(t_{1}\right), t_{0}\right) \\
\ldots \\
W_{\Delta}\left(\phi_{z}\left(t_{2 L}\right)\right.
\end{array}\right] z_{d} \equiv\left[\begin{array}{c}
W_{\Delta}\left(\phi_{z d}\left(t_{0}\right), t_{0}+\Delta\right) \\
W_{\Delta}\left(\phi_{z d}\left(t_{1}\right), t_{1}+\Delta\right) \\
\ldots \\
\cdots \\
W_{\Delta}\left(\phi_{z d}\left(t_{2 L}\right), t_{2 L}+\Delta\right)
\end{array}\right]
$$

the array of samples and its delayed version, respectively, where $\phi_{z}(\zeta)=\phi(y(\zeta), \zeta, t)$ and $\phi_{z d}(\zeta)=$ $\phi(y(\zeta), \zeta+\Delta, t)$. The $z, z_{d} \in \mathbb{R}^{\mathbb{N} \times(\mathbb{L}+)}$ are column stacked vectors. By applying to $z$ and $z_{d}$ the Discrete Fourier Transform (DFT), represented by the $\mathbf{F} \in \mathbb{R}^{(\mathbb{L}+\mathbb{1}) \mathbb{N} \times(\mathbb{L}+\mathbb{1}) \mathbb{N}}$ matrix, the corresponding $\mathcal{Z}=$ $\mathbf{F} z$ and $\mathcal{Z}_{d}=\mathbf{F} z_{d}$ approximate spectra are derived $\left({ }^{1}\right)$.

We use the word "approximate" since in general $W_{\Delta}(r(t), t)$, that represents a $\Delta=T / q$ window of $T$ periodic waveform, is not a periodic in the same interval.

However, if $W_{\Delta}(r(t+\Delta)-r(t), t)=0$ were satisfied, we would have $\mathcal{Z}_{d}=\mathbf{F} z_{d}=\mathbf{D}(\Delta) \mathcal{Z}$, where $\mathbf{D}(\Delta) \in \mathbb{R}^{(\mathbb{L}+\mathbb{1}) \mathbb{N} \times(\mathbb{L}+\mathbb{1}) \mathbb{N}}$ is the delay matrix computed by means of DFT. Now it is straightforward to write the equation

$$
\mathbf{F} z_{d}-\mathbf{D}(\Delta) \mathbf{F} z=0 \quad \rightarrow \quad z_{d}-\mathbf{F}^{-1} \mathbf{D}(\Delta) \mathbf{F} z=0
$$

that relates the $z_{d}$ vector of samples to the $z$ one. By definition (9) represents the new periodicity constraint. Finally the substitutions of (8) in (9) yields to the steady state problem

$$
\begin{gathered}
\Gamma\left(\widehat{y}_{s}\right)=\left[\begin{array}{c}
W_{\Delta}\left(\phi_{z d}\left(t_{0}\right), t_{0}+\Delta\right) \\
W_{\Delta}\left(\phi_{z d}\left(t_{1}\right), t_{0}+\Delta\right) \\
\ldots \\
W_{\Delta}\left(\phi_{z d}\left(t_{2 L}\right), t_{0}+\Delta\right)
\end{array}\right]- \\
\mathbf{F}^{-1} \mathbf{D}(\Delta) \mathbf{F}\left[\begin{array}{c}
W_{\Delta}\left(\phi_{z}\left(t_{0}\right), t_{0}\right) \\
W_{\Delta}\left(\phi_{z}\left(t_{1}\right), t_{1}\right) \\
\ldots \\
\cdots \\
W_{\Delta}\left(\phi_{z}\left(t_{2 L}\right), t_{2 L}\right)
\end{array}\right]=0
\end{gathered}
$$

whose unknowns are the $y\left(t_{0}\right), y\left(t_{1}\right), \ldots, y\left(t_{2 L}\right)$ initial conditions which are column staked into the $\widehat{y}_{s} \in$

$(+) \times$ vector. Equation (10) is well formed since it involves $2 L+1$ unknowns in $2 L+1$ independent equations and ensures that $W_{\Delta}(r(t+\Delta)-r(t), t)=0$, at least at the sampling time instants; note that this does not necessarily imply that $r(t)=0$.

\section{An ILlustrative EXAMPLE}

Consider the scalar equation (11) modelling a nonautonomous circuit driven by $P$ distinct sinusoidal sources

$$
\tau \frac{d y}{d t}(t)+y(t)=E+\sum_{p=0}^{P} \frac{a_{p}}{2}\left(e^{j 2 \pi \nu_{p} t}-e^{-j 2 * \pi \nu_{p} t}\right)
$$

where $\tau \in \mathbb{R}^{+}, E \in \mathbb{R}$ and $a_{p} \in \mathbb{R}, \nu_{p} \in \mathbb{R}^{+}$, with $\nu_{p}<\nu_{p+1}$, are the amplitude and the angular frequencies of the $P$ sources, respectively. The solution of (11) is

$$
\begin{gathered}
y(t)=\phi\left(y\left(t_{0}\right), t_{0}, t\right)=\widehat{\kappa} e^{-t / \tau}+E+ \\
\sum_{p=0}^{P}\left(\varrho_{p} e^{j 2 \pi \nu_{p} t}+\varrho_{p}^{*} e^{-j 2 \pi \nu_{p} t}\right)
\end{gathered}
$$

${ }^{1}$ Since $y(t)$ is not, in general, a scalar function, the $\mathbf{F}$ matrix must be organised so that DFT acts on each component of the $y(t)$ function. Moreover $\mathbf{F}$ is real since real and imaginary parts are on separate rows. 
where $\phi\left(y\left(t_{0}\right), t_{0}, t\right)$ is the state transition function and $\rho_{p}=\frac{a_{p}\left(1-j \tau 2 \pi \nu_{p}\right)}{2+2\left(\tau 2 \pi \nu_{0}\right)^{2}}$. Being $y\left(t_{0}\right)$ the initial condition, from (12) we have

$$
\widehat{\kappa} e^{-t_{0} / \tau}=y\left(t_{0}\right)-E-\sum_{p=0}^{P}\left(\rho_{p} e^{j 2 \pi \nu_{p} t_{0}}-\rho_{p}^{*} e^{-j 2 \pi \nu_{p} t_{0}}\right)
$$

the steady state condition for equation (11) is met when $\widehat{\kappa}=0$ and the steady state solution is

$$
y_{s}(t)=E+\sum_{p=0}^{P}\left(\varrho_{p} e^{j 2 \pi \nu_{p} t}+\varrho_{p}^{*} e^{-j 2 \pi \nu_{p} t}\right)
$$

The $r(t)$ function is represented by the $\widehat{\kappa} e^{-t / \tau}$ term that depends on the initial condition $y\left(t_{0}\right)$. Now by adopting as $w(\tau, t)$ the order 1 Hanning windowing function and applying the resulting $W_{\Delta}(\zeta(t), t)$ operator to (12) at the generic time instant $t_{n}$ we have

$$
z\left(t_{n}\right)=\underbrace{\int_{t_{n}}^{t_{n}+\Delta} \overbrace{\frac{\pi}{2 \Delta} \sin \left(\frac{\pi}{\Delta}\left(\tau-t_{n}\right)\right)}^{w(\tau, t)} \phi\left(y\left(t_{n}\right), t_{n}, \tau\right) d \tau}_{W_{\mathrm{HA}}(\zeta(t), t)}
$$

From (13) we see that

$$
\begin{gathered}
\lim _{(\Delta / \tau) \rightarrow 0} \frac{\widehat{\kappa}}{2+2\left(\frac{\Delta}{\tau \pi}\right)^{2}} e^{-t_{n} / \tau}\left(e^{-\Delta / \tau}+1\right)= \\
\widehat{\kappa} e^{-t_{n} / \tau} \equiv \widehat{\kappa}_{a} \\
\lim _{\left(\Delta \nu_{p}\right) \rightarrow \infty} \frac{1}{2-2\left(\Delta \nu_{p}\right)^{2}}\left[\left(e^{j 2 \pi \nu_{p} \Delta}+1\right) \varrho_{p} e^{j 2 \pi \nu_{p} t_{n}}+\right. \\
\left.\left(e^{-j 2 \pi \nu_{p} \Delta}+1\right) \varrho_{p}^{*} e^{-j 2 \pi \nu_{p} t_{n}}\right]=0
\end{gathered}
$$

The above limits show that if the integration time interval $\Delta$ is suitably chosen with respect to the $\tau$ natural frequency and to the $\nu_{p}$ frequencies of the sources, the integral (13) acts like a low pass filter that attenuates the amplitude of some "high frequency" components of the spectrum of $y(t)[1]$. Equation (13) allows us to conveniently reconstruct the samples at the time instants $t_{1}, t_{2}, \ldots, t_{2 L}$ of the $W_{\Delta}(y(t), t)$ function that we assume "slowly varying" and approximately characterised by a spectrum composed of the finite number $L$ of harmonics that lie well beyond $\nu_{0}$. We need thus $2 L+1$ samples in the $T$ period to completely reconstruct $W_{\Delta}(r(t), t)$. For simplicity suppose that $L=1$, thus we pick three time instants in the $T$ period: $t_{0}=0, t_{1}=T / 3$ and $t_{2}=2 T / 3$. The $z\left(t_{0}\right), z\left(t_{1}\right)$ and $z\left(t_{2}\right)$ samples are computed by means of the integral (13) and as described in section II, the components of the $Z(\widehat{f})$ corresponding spectrum are derived through the Fourier transform. In our simple case they are the DC component denoted by $Z_{\mathrm{DC}}$ and the component at the $\widehat{\nu}=1 / T$ fundamental frequency, denoted by $Z_{1}=Z_{1}^{\mathrm{R}}+j Z_{1}^{\mathrm{I}} \in$. In compact form we have

$$
\overbrace{\left[\begin{array}{c}
Z_{D C} \\
Z_{1}^{R} \\
Z_{1}^{I}
\end{array}\right]}^{\mathcal{Z}}=\mathbf{F} \overbrace{\left[\begin{array}{c}
W_{\Delta}\left(y(t), t_{0}\right) \\
W_{\Delta}\left(y(t), t_{1}\right) \\
W_{\Delta}\left(y(t), t_{2}\right)
\end{array}\right]}^{z}
$$

where $\mathbf{F}$ is the Fourier matrix. From (16), we can write $W_{\Delta}(y(t), t)=Z_{D C}+Z_{1}^{R} \cos (2 \pi \widehat{\nu} t)+Z_{1}^{I} \sin (2 \pi \widehat{\nu} t)$ that is, we can reconstruct the $W_{\Delta}(y(t), t)$ function in every time instant. In particular we are able to compute $W_{\Delta}(y(t), t)$ in three new time instants delayed by the same $\Delta$ interval with respect to $t_{0}, t_{1}$ and $t_{2}$. In matrix form, we have

$$
z_{d}=\underbrace{\left[\begin{array}{ccc}
1 & \cos \left(2 \pi \widehat{\nu}\left(t_{0}+\Delta\right)\right) & \sin \left(2 \pi \widehat{\nu}\left(t_{0}+\Delta\right)\right) \\
1 & \cos \left(2 \pi \widehat{\nu}\left(t_{1}+\Delta\right)\right) & \sin \left(2 \pi \widehat{\nu}\left(t_{1}+\Delta\right)\right) \\
1 & \cos \left(2 \pi \widehat{\nu}\left(t_{2}+\Delta\right)\right) & \sin \left(2 \pi \widehat{\nu}\left(t_{2}+\Delta\right)\right)
\end{array}\right]}_{\mathbf{F}_{d}^{-1} \mathbf{D}(\Delta)} \mathcal{Z}
$$

Now, by substituting (16) in (17) we have

$$
z_{d}=\mathbf{F}_{d}^{-1} \mathbf{D}(\Delta) \mathcal{Z}=\mathbf{F}_{d}^{-1} \mathbf{D}(\Delta) \mathbf{F} z
$$

The entries of the $z_{d}$ vector in (18) are derived from those of $z$ but, as said in section II, they can be also computed through (13) that represents the $W_{\Delta}(y(t), t)$ operator, by suitably integrating (12) along the interval $\left[t_{n}+\Delta, t_{n}+2 \Delta\right]$ starting from the same $y\left(t_{n}\right)$ initial conditions adopted to compute $z$. In other words the entries of both $z$ and $z_{d}$ depend on the $y\left(t_{0}\right), y\left(t_{1}\right)$ and $y\left(t_{2}\right)$ initial conditions. Once more this is evidenced by substituting (13) in (18)

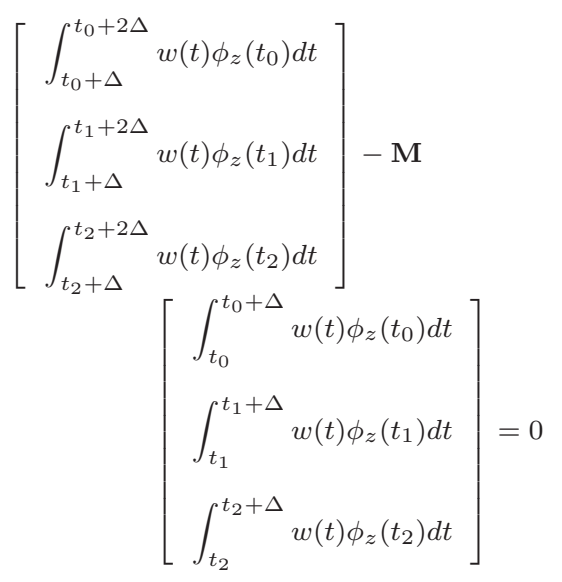

where $\mathbf{M}=\mathbf{F}_{d}^{-1} \mathbf{D}(\Delta) \mathbf{F}$. Problem (19) is well posed since it is composed of three independent equations involving the three unknowns $y\left(t_{0}\right), y\left(t_{1}\right)$ and $y\left(t_{2}\right)$. If we assume an ideal low pass filtering which implies that limits (14) and (15) hold, we have

$$
\begin{gathered}
{\left[\begin{array}{c}
\widehat{\kappa}_{0} e^{-\Delta / \tau}+E \\
\widehat{\kappa}_{1} e^{-\Delta / \tau}+E \\
\widehat{\kappa}_{2} e^{-\Delta / \tau}+E
\end{array}\right]-\mathbf{M}\left[\begin{array}{c}
\widehat{\kappa}_{0}+E \\
\widehat{\kappa}_{1}+E \\
\widehat{\kappa}_{2}+E
\end{array}\right]=0 \Longrightarrow} \\
(\mathbf{1}-\mathbf{M})\left[\begin{array}{c}
E \\
E \\
E
\end{array}\right]+\left(e^{-\Delta / \tau} \mathbf{1}-\mathbf{M}\right)\left[\begin{array}{c}
\widehat{\kappa}_{0} \\
\widehat{\kappa}_{1} \\
\widehat{\kappa}_{2}
\end{array}\right]=0
\end{gathered}
$$

where $\mathbf{1}$ is the identity matrix. Since the $\mathbf{M}$ matrix has a unit eigenvalue corresponding to the eigenvector whose entries are all ones, its multiplication by a constant vector gives the same vector as result; therefore $\widehat{\kappa}_{0}=\widehat{\kappa}_{1}=\widehat{\kappa}_{2}=0$, being $\left(e^{-\Delta / \tau} \mathbf{1}-\mathbf{M}\right)$ invertible. 


\section{Simulation Results}

The first circuit considered is the travelling waveform amplifier shown in fig. 3a; it is driven by the signal $\mathrm{v}_{\text {in }}(t)=v_{o}\left(\sin \left(2 \pi f_{1} t\right)+\sin \left(2 \pi f_{2} t\right)\right)$, where $v_{o}=45 \mathrm{mV}, f_{1}=100 \mathrm{MHz}$ and $f_{2}=102 \mathrm{MHz}$. The period of the resulting steady state waveforms is $500 \mathrm{~ns}$ (mcm of $1 / f_{1}$ and $1 / f_{2}$ ).

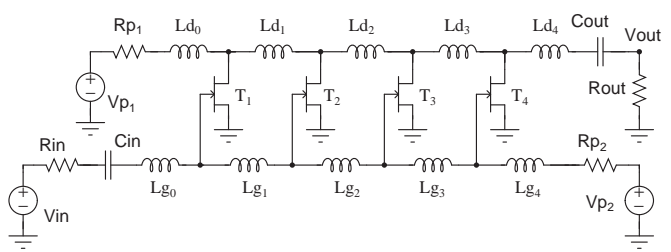

Fig. 3a. The schematic of the travelling waveform amplifier.

A portion of the periodic waveform at the output of the amplifier is shown in fig. 3b; we see that the waveforms computed by the harmonic balance method (HB) and the proposed method (MTS) basically overlap along each time window.

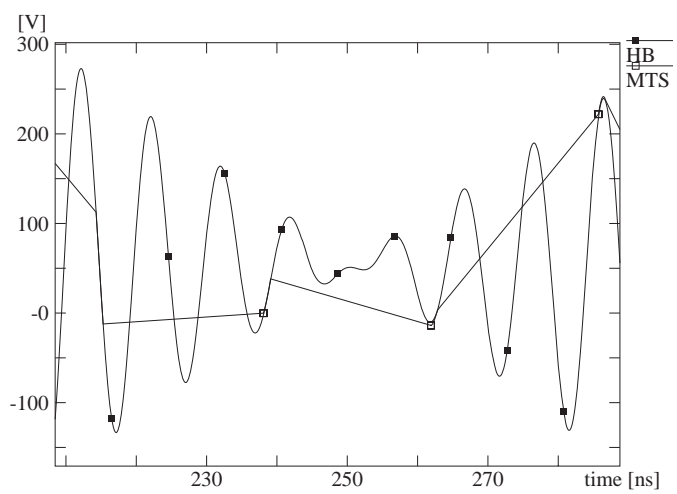

Fig. 3b. A portion of the periodic waveforms at the output of the travelling waveform amplifier.

Locations of time windows employed by MTS (total number equal to 21, corresponding to 10 harmonics, each 1 ns wide) can be easily found since they are "connected" by segments in fig. 3 b.

The second circuit considered is the mixer shown in fig. 4a. The local oscillator driving the mixer is represented by the $\mathrm{V}_{\text {lo }}$ independent voltage source that generates the sinusoidal waveform $0.1 \sin \left(2 \pi f_{\mathrm{LO}} t\right)$, where $f_{\mathrm{LO}}=1 \mathrm{GHz}$. The RF input is modelled by the Vrf voltage source that generates the signal $\sin \left(2 \pi f_{\mathrm{RF}} t\right)$, where $f_{\mathrm{RF}}=1 \mathrm{GHz}-100 \mathrm{MHz}$; the output filter of the mixer, composed of L2 and C2, is tuned at the $f_{\mathrm{IF}}=100 \mathrm{MHz}$. Figure $4 \mathrm{~b}$ shows the output waveforms of the mixer computed by HB and MTS. The MTS one has been obtained by employing 21 time windows each 100 ps wide. Once more we see a good agreement between the waveform computed by $\mathrm{HB}$ and the MTS one, even though, in this case, there is a certain phase shift between them.

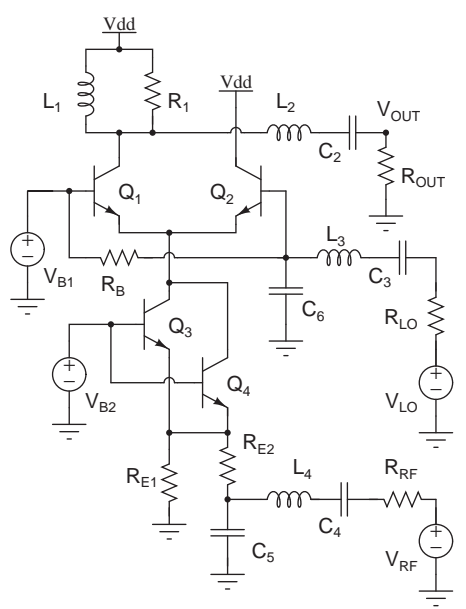

Fig. 4a. The schematic of the mixer.

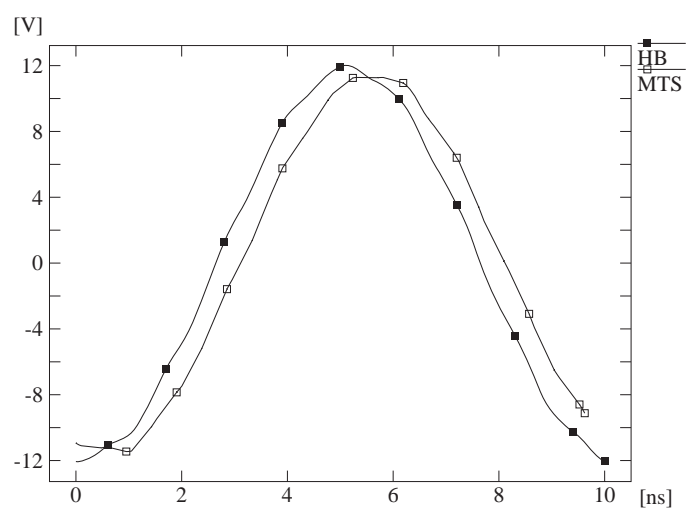

Fig. 4b. The Vout output waveform of the mixer.

\section{COnClusions And FUture WORK}

The numerical algorithm presented in this paper can be considered as the first version of an approach to simulate, in the time domain, non-autonomous circuits driven by two tone at different frequencies. As future work, we are going to develop a new version of the algorithm that removes the drawback mentioned in the introduction and increases numerical robustness, since the current version can lead, in some cases, to ill-conditioned or even singular matrices.

\section{REFERENCES}

[1] A. Brambilla, P. Maffezzoni, "Envelope Following Method to Compute Steady State Solutions of Electrical Circuits", IEEE Trans. on CAS-I, Vol. 50, No. 3, March 2003, pp. 999-1008.

[2] K. Kundert, J. White, A. Sangiovanni-Vincentelli, "A Mixed Frequency-Time Approach for Distortion Analysis of Switching Filter Circuits", IEEE Journal of Solid-State Circuits, Vol. 24, No. 2, April 1989, pp. 443-451.

[3] F. J. Harris, "On the Use of Windows for Harmonic Analysis with the Discrete Fourier Transform", Proceedings of the IEEE, Vol. 66, No. 1, January, 1978, pp. 51-84.

[4] J. Roychowdhury, "Analysing Circuits with Widely Separated Time Scales Using Numerical PDE Methods", IEEE Trans. on Circuits and Systems I, Vol. 48, No. 5, May 2001, pp. 578-595.

[5] J. Roychowdhury, "A Time-domain RF Steady-State Method for Closely Spaced Tones", Proceedings of DAC, June 10-14, 2002, New Orleans, LU, USA, pp. 510-513. 